Archivos de Coloproctología, 2019; 3(3):05-47

DOI: https://doi.org/10.26754/ojs_arcol/arch_colo.201934001

\title{
Actualización sobre el estoma de protección tras resección anterior baja 0 ultrabaja por cáncer de recto. Revisión sistemática y nuevas tendencias.
}

\author{
Gámez Córdoba ME
}

Cirujano general y del Aparato Digestivo. Unidad Cirugía Barcelona. Hospital Universitario Quirón Dexeus. Calle Sabino Arana 5-19, 08029, Barcelona, España.

\section{RESUMEN}

El estándar para el tratamiento del cáncer de recto es la resección anterior baja o ultrabaja con escisión total del mesorrecto. Técnicas cada vez menos invasivas unidas a un mayor interés por la preservación de esfínteres han llevado a realizar resecciones rectales con anastomosis cada vez más próximas al margen anal. Una de las complicaciones más importantes y más temidas por sus graves consecuencias es la fuga anastomótica. Con intención de prevenir la dehiscencia anastomótica o aliviar sus consecuencias se realizan tradicionalmente estomas derivativos tras la resección rectal con anastomosis de riesgo. Sin embargo el estoma de protección presenta morbilidad asociada, afecta a la calidad de vida del paciente, y precisa una nueva intervención para la reconstrucción del tránsito, que asocia a su vez una morbi-mortalidad significativa. Incluso uno de cada 4-6 estomas queda sin cerrar. Por todo ello el papel de los estomas de protección sigue siendo controvertido, sin encontrar respuestas con suficiente evidencia en la literatura.

Este estudio tiene como objetivo hacer una revisión de la literatura para establecer el rol actual del estoma de protección. Además analizamos la corriente que boga por el cierre precoz del estoma (en los primeros 15 días tras la cirugía) y las alternativas a los estomas de protección tradicionales que han 


\section{Archivos de Coloproctología}

Archivos de Coloproctología, 2019; 3(3):05-47

DOI: https://doi.org/10.26754/ojs_arcol/arch_colo.201934001

aparecido en los últimos años, como la ileostomía virtual, el tubo ileal y el dispositivo de derivación fecal.

Palabras clave: estomas de protección, cáncer de recto, fuga anastomótica

\section{ABSTRACT}

Low or ultralow anterior resection with total mesorectal excision is the standard treatment for rectal cancer. Less invasive procedures and higher rates of sphincter- sparing preservation have resulted in an increase in the use of very low anastomosis in rectal resections. The most feared and devasting complication is the anastomotic leak. In order to prevent the anastomotic leak or to alleviate its consequences, derivative stomas are traditionally performed after rectal resection with risk anastomosis. However, diverting stomas are associated with morbidity, affects patients' quality of life and a new surgical procedure is necessary for stomal reversal, which in turn associates a significant morbidity and mortality. Moreover one in every 4-6 temporal stomas won`t be closed. Therefore, the role of protective stoma remains controversial, with no evidence in the literature.

The aim of this study is to review the literature to establish the role of diverting stomas. In addition, we analyze the current trend for the early closure of the stoma (in the first 15 days after surgery) and new procedures recently reported, such as virtual ileostomy, temporary tube stoma and fecal diverting device.

Keywords: Rectal cancer, Diverting stomas, anastomotic leak. 


\section{Archivos de Coloproctología}

Archivos de Coloproctología, 2019; 3(3):05-47

DOI: https://doi.org/10.26754/ojs_arcol/arch_colo.201934001

\section{INTRODUCCIÓN}

Actualmente el estándar para el tratamiento del cáncer de recto es la resección anterior baja (RAB) o ultrabaja (RAUB) con escisión total del mesorrecto (ETM). En los últimos 20 años la cirugía colorrectal se ha desarrollado hacia técnicas mínimamente invasivas con mayor énfasis en la preservación de esfínteres y menores tasas de estomas permanentes, lo que ha llevado a un incremento de anastomosis colorrectales cada vez más próximas al margen anal [1]. Además, la estandarización de la ETM se asocia con un incremento del riesgo de fuga anastomótica (FA), que constituye una de las complicaciones más graves en cirugía colorrectal con un gran impacto en la morbilidad y mortalidad postoperatorias. La FA puede afectar incluso a los resultados oncológicos disminuyendo el control local y la supervivencia a largo plazo [2-4].

\section{Riesgo de fuga anastomótica.}

Cuando se produce una FA pueden aumentar la morbilidad, mortalidad y estancia hospitalaria, y se produce un efecto negativo sobre la calidad de vida del paciente a largo plazo [5].

Se considera la tasa de FA como un indicador de calidad en cirugía colorrectal. Las tasas de FA reportadas son variables, según se tengan en cuenta o no las dehiscencias asintomáticas diagnosticadas por radiología. La media se sitúa en torno al $11-15 \%$ [1].

Es difícil predecir que pacientes pueden presentar FA, incluso para cirujanos experimentados. Y por el contrario, la FA puede producirse incluso cuando la anastomosis es técnicamente impecable y no existen factores de riesgo identificables. El riesgo de FA se puede deducir atendiendo a factores técnicos y a las características del paciente. Gran cantidad de artículos en la literatura han señalado como posibles factores de riesgo el sexo masculino, desnutrición, patología cardiovascular, tratamiento con corticoides preoperatorio, consumo de 
Archivos de Coloproctología, 2019; 3(3):05-47

DOI: https://doi.org/10.26754/ojs_arcol/arch_colo.201934001

tabaco o de alcohol, trasfusión perioperatoria, edad avanzada, obesidad y la radiación pélvica [1]. El estudio retrospectivo de Kang et al. sobre 72.055 pacientes mostró como factores de riesgo independientes de FA en el análisis multivariante: malnutrición como el principal factor (OR 2.81), seguido por las alteraciones hidroelectrolíticas (OR 1.79), el sexo masculino (OR 1.49), y la presencia de estoma de protección.[6] El efecto de la radiación es controvertida según diferentes estudios. [7-8]. Por otro lado la fuerte relación entre los tumores bajos y las anastomosis colorrectales bajas y la alta tasa de FA está bien establecida y confirmada por el estudio de Bertelsen et al. que muestra que los tumores por debajo de $10 \mathrm{~cm}$ desde el margen anal tienen un mayor riesgo de FA cuanto más se acercan al margen anal [9].

\section{Evaluación de anastomosis de riesgo.}

Para minimizar el riesgo de complicaciones anastomóticas en el postoperatorio se emplean diversas técnicas para asegurar la integridad de la misma. Se incluyen la comprobación de estanqueidad mecánica, técnicas de visualización endoscópica, y más recientemente la tecnología de evaluación de microperfusión.

Las pruebas de estanqueidad mecánicas incluyen la infusión de aire y fluidos (solución salina o azul de metileno). Estos test tienen la limitación de no visualizar la luz. Se comprueba la anastomosis ocluyendo el margen proximal, e introduciendo a través del extremo distal aire o fluido que rellenan el espacio endoluminal comprobando la existencia de fugas. En una revisión sistemática de dos ensayos clínicos comparando la comprobación con aire versus no comprobación demuestra un incremento de riesgo de FA en el grupo no comprobado (5.8\% FA en grupo TEST vs $16 \%$ FA en grupo NO TEST, $p<0.05$ ) $[1,10,11]$. 


\section{Archivos de Coloproctología}

Archivos de Coloproctología, 2019; 3(3):05-47 DOI: https://doi.org/10.26754/ojs_arcol/arch_colo.201934001

El empleo de la endoscopia intraoperatoria permite una visión directa endoluminal de la anastomosis e incluso la posibilidad de una intervención terapéutica en caso de comprobarse un defecto. En una revisión sistemática de 5 estudios no aleatorizados no se muestra diferencia en la tasa de FA entre los grupos test / control (7.7\% FA en Test +, 7.3\% FA en Test -, 5.9\% FA en grupo NO Test, $p$ 0.30)[12,13].

Las técnicas de comprobación de microperfusión para evaluar la anastomosis colorrectal están ganando popularidad. La tecnología permite el análisis de la perfusión tisular pudiendo detectar zonas de isquemia y realizar una reconstrucción de la anastomosis en caso necesario.

Las comprobaciones intraoperatorias de la anastomosis colorrectal continúan evolucionando con el avance de la tecnología quirúrgica. Mientras las pruebas mecánicas y endoscópicas parecen tener moderados beneficios en la disminución de FA, hay una creciente evidencia de que las técnicas de microperfusión y tintes auto fluorescentes pueden tener un mayor impacto en la reducción de las complicaciones anastomóticas [1].

\section{Consecuencias de la fuga anastomótica.}

La FA incrementa la mortalidad tras la cirugía colorrectal debido a sepsis pélvica y peritonitis con tasas publicadas de hasta el $22 \%$. Además en varios artículos se demuestra la influencia de la FA en los resultados oncológicos a medio-largo plazo, con un incremento de la recurrencia local y disminución de la supervivencia. Estas consecuencias han conducido a la doctrina quirúrgica tradicional que defiende la derivación fecal para paliar las devastadoras consecuencias de una dehiscencia anastomótica $[1,14,15,16]$.

\section{Estomas de protección.}




\section{Archivos de Coloproctología}

Archivos de Coloproctología, 2019; 3(3):05-47

DOI: https://doi.org/10.26754/ojs_arcol/arch_colo.201934001

Con intención de prevenir la aparición de fugas anastomóticas y reducir su morbimortalidad en anastomosis pélvicas de alto riesgo se han realizado tradicionalmente estomas de protección (EP) como medio de derivación fecal. Los EP son ampliamente empleados en cirugía colorrectal aunque en los últimos años su efectividad e indicaciones son motivo de debate con diversidad de resultados y opiniones en la literatura.

Las tasas de EP en pacientes con RAB son variables. Algunos estudios multicéntricos de cohortes muestran una incidencia de EP tras RAB entre el 32.3 y $42.1 \%$ [17-20]. Un gran estudio multicéntrico [6] de 2013 que recoge los datos de

72.055 sometidos a RAB en el estado de California (USA) revela diferencias según el tipo de cirugía. Según sus resultados de los pacientes intervenidos con RAB abierta el $15.89 \%$ recibió EP en la cirugía inicial, y de los pacientes con $R A B$ laparoscópica sólo el $1.17 \%$ recibió estoma de protección inicialmente. Probablemente estos resultados están artefactuados por un sesgo de selección, al influir las características del paciente en la elección de la técnica quirúrgica. Es posible que los pacientes intervenidos por vía abierta presenten a priori un mayor riesgo, por lo que presentan mayor tasa de EP de presumible mayor riesgo [6]. En un estudio retrospectivo sobre 203 pacientes con RAB publicado este mismo año la tasa de EP reflejada es del 69\% [21] coincidiendo con los resultados de otras publicaciones que corroboran que los estomas de protección siguen siendo ampliamente utilizados en la mayoría de pacientes sometidos a una RAB [22-24].

\section{Indicaciones del estoma de protección.}

En la mayoría de los casos la decisión de confeccionar un estoma derivativo depende de la experiencia del cirujano. Un estudio retrospectivo japonés realizado sobre 1014 pacientes estimó como factores más relacionados con la 
Archivos de Coloproctología, 2019; 3(3):05-47

DOI: https://doi.org/10.26754/ojs_arcol/arch_colo.201934001

elaboración de un EP: sexo masculino, tumor de gran tamaño, tumores avanzados (T3-T4), afectación ganglionar, quimio y/o radioterapia neoadyuvantes, localización baja del tumor, anastomosis baja, sangrado masivo intraoperatorio, ligadura alta de la arteria mesentérica inferior, linfadenectomía lateral, cirugía abierta, rodete anastomótico incompleto y test de estanqueidad de anastomosis positivos [18]. En la reunión de consenso polaca se incluyen como indicaciones para el EP: anastomosis a $<3 \mathrm{~cm}$ de la línea pectínea, neoadyuvancia, inmunosupresión, test de estanqueidad positivo y/o rodetes de anastomosis incompletos, IMC < 19, ASA III/IV y dificultados intraoperatorias significativas (pelvis estrecha) [25]. En una encuesta realizada a los miembros de la Sociedad Americana de Cirujanos Colorrectales la irradiación pélvica, los niveles bajos de albúmina/desnutrición y el uso de corticoides/inmunosupresión fueron los factores considerados más importantes en la decisión de confeccionar un EP tras una anastomosis no óptima a nivel de recto medio-bajo [26].

\section{Evidencia sobre el estoma de protección.}

A partir del año 2000 empieza a debatirse el papel del EP en los foros de cirugía colorrectal tras varias publicaciones que cuestionan la necesidad y los beneficios del EP sistemático en los pacientes con anastomosis bajas [1,27-31]. Sigue existiendo controversia respecto al efecto protector del EP sobre el riesgo de FA. Si bien es reconocido que el EP disminuirá la magnitud de la sepsis y morbilidad asociadas a las FA una vez que esta se produce, existen estudios que aseguran no encontrar influencia sobre la incidencia de fugas, tasa de reintervenciones y complicaciones asociadas tras la RAB sin EP [21,32].

Hay pocos estudios que comparen específicamente las complicaciones globales en pacientes tratados con y sin EP tras RAB, y éstos generalmente presentan resultados controvertidos. 


\section{Archivos de Coloproctología}

Archivos de Coloproctología, 2019; 3(3):05-47

DOI: https://doi.org/10.26754/ojs_arcol/arch_colo.201934001

Revisando las publicaciones más relevantes (últimos 5 años, y anteriores con mayor evidencia) encontramos algunos estudios y meta-análisis que analizan la influencia del estoma derivativo en la tasa e impacto de FA tras RAB por cáncer de recto.

\section{Estudios no aleatorizados.}

El estudio retrospectivo multicéntrico de Gastinger et al. compara 2729 pacientes con RAB de los cuales 881 recibieron EP y 1848 no recibieron EP. Los resultados no encuentran diferencias en cuanto a la tasa de FA entre los grupos, no obstante la tasa de reintervenciones es significativamente menor en el grupo de pacientes con EP $(p<0.01)$ [20]. En Suecia se realiza otro gran estudio multicéntrico retrospectivo publicado por Matthiessen et al. en el que se realizan EP a 432 de 6833 paciente que tampoco observa diferencias en la tasa de FA (15\% EP vs $12 \%$ No EP) [33].

Entre los estudios que defienden el uso rutinario del EP encontramos un estudio multicéntrico retrospectivo que incluye 482 pacientes de 75 centros alemanes y demuestra un efecto protector del EP sobre la tasa de FA [34], y otro estudio danés que incluye 924 pacientes tras ETM que demuestra una reducción de la tasa de FA en pacientes con drenaje pélvico y estoma de protección $(p<0.01)$ [35].

En 2013 Nurkin et al. realizan un estudio retrospectivo multicéntrico que incluye 1791 pacientes intervenidos con RAB procedentes de 211 centros. 1266 pacientes presentaban anastomosis pélvicas bajas. De ellos 606 recibieron EP y 660 no. No se objetivaron diferencias en cuanto a las complicaciones de la herida quirúrgica, sepsis o incidencia de shock séptico entre los grupos. Sin embargo, en el grupo de pacientes con EP se observó mayor incidencia de insuficiencia renal (OR 3.67, p < 0.05). En 525 pacientes con anastomosis coloanal sin estoma, se observó un aumento significativo de riesgo de sepsis (OR 
Archivos de Coloproctología, 2019; 3(3):05-47

DOI: https://doi.org/10.26754/ojs_arcol/arch_colo.201934001

2.47, $p<0.05$ ), shock séptico (OR 6.29, p < 0.05), y reintervención (OR 7.11, p $<0.05)$. Un análisis multivariante demostró que los pacientes con anastomosis colo-anal sin estoma presentaban un aumento considerable de riesgo de complicaciones postoperatorias graves [36].

También publicado en 2013, el estudio multicéntrico de Kang et al. recoge los datos de 72.055 pacientes sometidos a RAB en California. Los resultados revelan diferencias en la tasa de FA según el tipo de cirugía. En RAB abierta la incidencia de FA fue mayor que en la RAB laparosópica, independientemente del EP. En Resecciones laparoscópicas y abiertas con EP también mostraron mayor tasa de FA que los intervenidos por ambas vías de abordaje sin EP (15.97\% vs $13.25 \%)$. Probablemente el sesgo de selección al influir las características del paciente en la elección de la técnica y del uso del EP artefactúa los resultados [6].

Uno de los estudios publicados más recientemente compara 203 pacientes tras RAB, de los cuales 140 tuvieron EP inicialmente (69\%). Los resultados no revelan diferencias significativas en cuanto a la tasa de FA/sepsis abdominal, reingresos o mortalidad. Si se encontró una reducción del número de reintervenciones en el grupo con EP (EP 7.9\% vs No EP 20.6\%; OR 0.33 [IC 95\% 0.14-0.78]). Sin embargo, la

estancia hospitalaria inicial fue mayor en los pacientes con EP (EP 13.0 vs No EP 6.9, p 0.005). Tras un análisis de regresión logística el estoma de protección aparece como un factor de riesgo independiente sobre el riesgo de complicaciones postoperatorias tras RAB (OR 2.62, IC 95\% 1.19-5.78; p 0.02), junto con un ASA $\geq 3$ (OR 2.39, IC 95\% 1.15-4.98; p 0.02) [21].

También apuntan a un aumento de complicaciones postoperatorias globales en los pacientes portadores de EP los resultados del estudio de Belkin et al. que encuentra un incremento en la estancia hospitalaria (6.7 vs 3.1 días, $p$ 0.0003), más complicaciones peroperatorias ( $57.1 \%$ vs $13.9 \%$, $p$ 0.0006), y una mayor 


\section{Archivos de Coloproctología}

Archivos de Coloproctología, 2019; 3(3):05-47

DOI: https://doi.org/10.26754/ojs_arcol/arch_colo.201934001

tasa de reingresos o visitas en urgencias en los siguientes 60 días a la cirugía (38.1\% vs 5.6\%, p 0.002) en los pacientes portadores de EP [37].

Los estudios no aleatorizados presentan un importante sesgo de selección que favorece al grupo de cirugía sin estomas. Este sesgo es inherente ya que estos estudios dependen de la experiencia del cirujano, que toma la decisión de crear un estoma de forma selectiva en aquellos pacientes que pueden presentar factores de riesgo de complicación de la anastomosis.

\section{Estudios aleatorizados}

El primer gran estudio aleatorizado (EA) focalizado en el estoma protección es el publicado en 2007 por Matthiessen et al. Presenta los resultados de un análisis de 234 pacientes, aleatorizados en 2 brazos: Estoma (n 116) y No estoma ( $\mathrm{n}$ 118). La tasa de fuga anastomótica observada fue $10.3 \%(12 / 116)$ en grupo Estoma y $28 \%(33 / 118)$ en grupo No estoma, con una diferencia estadísticamente significativa $(p<0.05)$. Como es de esperar, el número de reintervenciones es significativamente mayor en el grupo de pacientes sin estoma (30/118; 25.4\%) en comparación con el grupo con estoma (10/116; 8.6\%), p < 0.05 [38]. En 2008 aparece un nuevo EA publicado por Chude et al. que incluye 256 pacientes, aleatorizados en 2 grupos: No estoma ( $n$ 120) y estoma (n 136). Las FA observadas fueron mayores en el grupo de no estoma (NE 12/120(10\%) vs E 3/136(2.2\%)). Además, en el grupo de NO estomas fueron necesarias 2 reintervenciones, mientras que ningún paciente del grupo con estomas necesitó reintervención [39]. En los estudios aleatorizados parece demostrarse el efecto protector del estoma derivativo sobre la tasa de FA y de reintervenciones.

META-ANÁLISIS Y REVISIONES SISTEMÁTICAS 


\section{Archivos de Coloproctología}

Archivos de Coloproctología, 2019; 3(3):05-47 DOI: https://doi.org/10.26754/ojs_arcol/arch_colo.201934001

En 2008 se publica una revisión sistemática de estudios retrospectivos sobre la ileostomía de protección incluyendo estudios que la realizaban de forma sistemática, de forma selectiva y que rechazaban el uso de estomas. En todos ellos existe un sesgo de selección importante, por lo que no se pueden extraer conclusiones con suficiente evidencia. Sin embargo, a pesar del sesgo que favorece al grupo de No estoma, los pacientes con estoma no presentaron peores resultados. Este mismo grupo realiza un meta-análisis de los estudios prospectivos aleatorizados presentes hasta ese momento. Los resultados mostraron un menor riesgo de riesgo de $\mathrm{FA}(Z 3.65, \mathrm{p}<0.01)$ y menor riesgo de reintervención $(Z 0.05, p<0.01)$ en el grupo de pacientes con estoma, de forma significativa [40]. No obstante la mortalidad no mostró diferencias entre los grupos. Estos resultados concuerdan con otro metaanálisis realizado posteriormente y publicado por Cochranne. Este estudio, que toma los datos de $6 \mathrm{EA}$, analiza los datos de 648 pacientes. Con respecto a los controles ( $\mathrm{n} 316$ ), el uso de EP ( $n$ 332) se asoció con menor riesgo de FA (RR 0.33, IC 95\% 0.21 0.53), y menos reintervenciones urgentes (RR 0.23, IC 95\% 0.12-0.42). No se observaron diferencias en cuanto a la mortalidad (RR 0.58, IC 95\% 0.14-2.33) [41].

El metaanálisis publicado por el equipo de Chen et al. en 2012, que recoge 878 pacientes de $5 \mathrm{EA}$, estima una reducción del riesgo de FA con el EP (RR 0.34, IC 95\% 0.22-0.53), así como del riesgo de reintervención (RR 0.27, IC 95\% 0.161.48, $\mathrm{p}<0.00001)$. Este estudio lanza una advertencia ante la necesidad de estudios que analicen la influencia sobre la mortalidad a largo plazo y la calidad de vida [42].

En 2014 un nuevo meta-análisis intenta esclarecer el papel del EP en la RAB por cáncer de recto recogiendo los datos de 11 EA. En total se incluyen los datos de 5612 pacientes, 2868 de los cuales recibieron EP y 2744 no llevaron estoma. Los resultados apuntan que la ausencia de un EP se asocia a una mayor 


\section{Archivos de Coloproctología}

Archivos de Coloproctología, 2019; 3(3):05-47

DOI: https://doi.org/10.26754/ojs_arcol/arch_colo.201934001

incidencia de fuga anastomosis y reintervención con RR estimadas de 0.38 (IC $95 \%, \quad 0.30-0.48$, p <0.00001) y 0.37 (IR 95\%, 0.29-0.48, p <0.00001) respectivamente. El estudio concluye un beneficio estadísticamente significativo por parte del estoma derivativo en pacientes intervenidos con anastomosis bajas [43]. En 2015 el mismo grupo realiza un nuevo meta-análisis incluyendo 13 estudios que recogen los resultados de 8002 pacientes. Los resultados concuerdan con el estudio anterior y el EP se asocia con un menor riesgo de fuga anastomótica (RR 0.47, IC 95\% 0.33-0.68, p <0.00001) y de reintervención (RR 0.36, IC 95\% 0.28-0.46, p <.00001) [30].

Los meta-análisis corroboran el efecto protector del estoma sobre la tasa de FA y reintervenciones urgentes. Hasta ahora ningún EA o meta-análisis incluye datos en referencia al efecto sobre la supervivencia a largo plazo y calidad de vida relacionados con el estoma de protección.

\section{lleostomía vs colostomía.}

La ileostomía y colostomía en asa son las dos técnicas tradicionales empleadas para los EP [1]. Según las publicaciones, no existen diferencias significativas en cuanto a ileostomía o colostomía de protección. Según una revisión Cochrane sobre 5 EA a este efecto, la ileostomía en asa presenta menos riesgos de prolapso [44]. Los resultados y funcionalidad parecen similares. No obstante la ileostomía en asa parece el estoma de protección de elección, probablemente por su mayor facilidad de confección y reconstrucción, mejor efectividad de derivación, mayor distancia a la anastomosis, por producir menos olor y porque no presenta riesgo de lesionar la arcada vascular pericólica [45-46].

\section{Morbilidad del estoma de protección.}

A pesar de los reconocidos efectos beneficiosos del EP en la prevención de FA y su morbilidad asociada tras $\mathrm{RAB}$, la realización de un estoma es un gesto 


\section{Archivos de Coloproctología}

Archivos de Coloproctología, 2019; 3(3):05-47

DOI: https://doi.org/10.26754/ojs_arcol/arch_colo.201934001

quirúrgico que no está exento de complicaciones, que tiene un importante impacto en la calidad de vida del paciente y exigirá una nueva intervención quirúrgica para su reconstrucción, con lo cual no puede considerarse una decisión banal. La confección del estoma constituye normalmente el último paso de una intervención compleja, y a pesar de ser un procedimiento relativamente sencillo, es necesaria una ejecución meticulosa de la técnica quirúrgica para minimizar la morbilidad. Las complicaciones del EP reflejadas en los estudios varían del 10 al 70\% [1,21,47-51]. Esta variabilidad puede deberse en parte a la falta de una definición concreta de las complicaciones del estoma en la comunidad quirúrgica. Las complicaciones asociadas al estoma varían desde complicaciones menores, que requieren un tratamiento local, a complicaciones mayores con necesidad de reintervención, que ocasionan un incremento de la estancia hospitalaria y un retraso en la restitución del paciente a una vida activa $[1,52]$. La principal complicación referida por los pacientes es la irritación de la piel periestomal. Esta complicación no siempre es recogida como tal por el cirujano. En un estudio comparativo reciente los resultados reflejan una tasa de complicaciones relacionadas con el estoma del 19.3\% en los primeros 90 días, con un $2.9 \%$ de reintervenciones asociadas [21].

\section{Factores de riesgo de complicaciones del estoma.}

Los factores de riesgo de presentar complicaciones del estoma se dividen según su relación con el paciente o con la técnica quirúrgica. Los factores dependientes del paciente son la edad, género, BMI, estado nutricional, ASA, tratamiento con corticoides, cirugía oncológica, inmovilidad, hábito tabáquico, diabetes. En relación al procedimiento, los estomas realizados en ámbito de cirugía de urgencias y con una altura inferior al $\mathrm{cm}$ tienen mayor riesgo de presentar complicaciones [53]. A pesar de los avances en los procedimientos y manejo peroperatorio, las complicaciones estomales siguen siendo frecuentes. 


\section{Archivos de Coloproctología}

Archivos de Coloproctología, 2019; 3(3):05-47

DOI: https://doi.org/10.26754/ojs_arcol/arch_colo.201934001

\section{Complicaciones locales.}

Un estoma mal ubicado puede condicionar un incremento de la morbilidad. Existe suficiente evidencia sobre la relación negativa de una localización inadecuada del estoma sobre la esfera psicológica, imagen corporal y recuperación postoperatoria [54,55].

El marcaje preoperatorio es recomendable siempre que exista la posibilidad de realizar un estoma, tanto en cirugía electiva como en cirugía urgente. Como principio general el sitio adecuado para el estoma es aquel que no está en relación con prominencias óseas, pliegues o incisiones. Habitualmente este área corresponde a los 2/3 de la línea entre la espina ilíaca anterosuperior y el ombligo [56]. Para anticipar posibles problemas de localización se recomienda marcar y explorar a los pacientes en bipedestación, sedestación y decúbito supino. En pacientes obesos deben colocarse más craneales de lo habitual en zonas de la pared abdominal con una grasa subcutánea más delgada y que permitan mejor acceso y visualización de los dispositivos estomales [57].

\section{Complicaciones a corto plazo y largo plazo.}

Las complicaciones cutáneas periestomales varían desde leve irritación hasta grandes áreas de ulceración e infección. La adecuada localización y confección del estoma son las principales medidas para prevenir estas complicaciones. Presentan mayor riesgo los pacientes obesos en los que existe mayor dificultad para ajustar los dispositivos de estoma por los pliegues cutáneos. La falta de enfermero/a estomatoterapeuta se ha identificado como el principal factor de riesgo para desarrollar complicaciones dérmicas periestomales. Los pacientes deben ser educados en el cuidado del estoma, colocación y extracción de los dispositivos de forma adecuada, y reajuste de las medidas según la evolución del estoma y la pared abdominal [58-60]. 


\section{Archivos de Coloproctología}

Archivos de Coloproctología, 2019; 3(3):05-47

DOI: https://doi.org/10.26754/ojs_arcol/arch_colo.201934001

Una de las complicaciones más importantes y causa de numerosos reingresos hospitalarios es la deshidratación o alteraciones hidroelectrolíticas secundarias a las pérdidas excesivas por el estoma. La incidencia varía entre 1-16\% según los estudios publicados. No es infrecuente alcanzar débitos de hasta 2 litros diarios en el postoperatorio inicial ya que el edema intestinal disminuye la capacidad de absorción de líquidos en la mucosa. La hipopotasemia, la hipomagnesemia y la hipocalcemia son hallazgos comunes, y es relativamente frecuente la deshidratación grave que puede llegar a producir insuficiencia renal [60]. El momento de mayor riesgo es entre el $4^{\circ}$ y $8^{\circ}$ días postoperatorios. Entonces muchos de ellos ya han recibido el alta hospitalaria. Es necesaria una educación adecuada del paciente orientada a vigilar los débitos del estoma y ajustar la ingesta de líquidos para compensar las pérdidas. Además se debe enseñar que el vaciado frecuente de las bolsas (> 5 veces al día), la presencia de fugas, aparición de náuseas, mareos, malestar o fatiga, pueden ser signos de pérdida excesiva de líquidos. Los pacientes deben evitar los líquidos hipotónicos que causan hiponatremia, siendo preferible ingerir soluciones balanceadas en electrolitos y glucosa para el aporte hídrico [45]. En casos incontrolables se pueden emplear tratamientos adicionales como la loperamida o el difenoxilato [1].

La hernia paraestomal es una complicación temida y molesta a largo plazo. Su incidencia aumenta con el tiempo desde la intervención y puede alcanzar hasta el $15-40 \%$ de los ostomizados. Según la literatura la ubicación y el tipo de estoma no aumentan el riesgo de hernia paraestomal. Los factores de riesgo de presentar hernia paraestomal son similares a los factores de riesgo conocidos para otros tipos de hernias de pared abdominal. Se han relacionado ciertos factores de forma significativa con el riesgo de presentar hernia periestomal: diabetes, patología respiratoria, cirugía oncológica y colostomías terminales $[61,62]$. Las intervenciones para corregir las hernias paraestomales incluyen 


\section{Archivos de Coloproctología}

Archivos de Coloproctología, 2019; 3(3):05-47

DOI: https://doi.org/10.26754/ojs_arcol/arch_colo.201934001

reparación local, reubicación y reparación con malla. En estomas definitivos se ha incrementado el interés por el uso de las prótesis profilácticas.

Sin duda, el estoma supone un impacto en la calidad de vida el paciente, afectando a su esfera física, social y mental. Solo entre las publicaciones más recientes encontramos análisis de la influencia en la calidad de vida de los pacientes ostomizados. El estudio observacional multicéntrico sobre 17 centros alemanes que recoge los datos de 120 pacientes con EP tras RAB encuentra un efecto negativo significativo sobre la funcionalidad social y la sintomatología de tipo gastrointestinal, sin una influencia negativa significativa sobre la calidad de vida global de los pacientes [63]. Son necesarios programas de formación con un equipo de enfermería especializado en estomatoterapia para incrementar la calidad de vida de los paciente ostomizados. La revisión sistemática publicada por Danielsen et al. refleja que un programa y equipo especializados en la estomatoterapia incrementa la calidad de vida, la eficiencia en los cuidados del estoma, reduce la estancia hospitalaria y disminuye el impacto psicosocial [64].

\section{Timing del cierre del estoma de protección.}

El tiempo óptimo entre la creación y el cierre del EP es controvertido. Generalmente se considera adecuado un período de 8-12 semanas desde la primera cirugía. Este tiempo sería suficiente para permitir la recuperación del paciente, disminuir la densidad adherencial intraabdominal y permitir una resolución de los cambios inflamatorios y el edema en el abdomen y el estoma [65]. Según algunos autores un intervalo de tiempo inferior a 8 semanas incrementaría el riesgo de complicaciones y un tiempo superior es un factor predictivo negativo en los resultados [66-67]. Sin embargo cada vez más autores argumentan a favor de un cierre precoz del estoma en pacientes seleccionados, sin encontrar un aumento en la morbilidad. 


\section{Archivos de Coloproctología}

Archivos de Coloproctología, 2019; 3(3):05-47

DOI: https://doi.org/10.26754/ojs_arcol/arch_colo.201934001

Algunos trabajos han demostrado que el cierre precoz puede poner en riesgo el adecuado cumplimiento del tratamiento adyuvante debido a complicaciones como la fuga anastomótica o el síndrome de RAB. El Síndrome de RAB es una situación clínica que se produce tras la resección anterior baja, que puede afectar gravemente a la calidad de vida de los pacientes. Generalmente se manifiesta en los primeros meses tras el cierre del estoma y puede agravar los efectos secundarios de la quimioterapia, de nuevo resultando en un obstáculo para que los pacientes puedan recibir los ciclos necesarios de quimioterapia adyuvante [78].

El momento óptimo para el cierre del EP, asegurando una quimioterapia adyuvante completa, sigue siendo controvertido. A priori parece que lo mejor es reconstruir el tránsito lo antes posible, pero por otro lado el cierre puede resultar en más complicaciones asociadas que pueden poner en peligro la adecuada consecución del tratamiento adyuvante. En este sentido se propuso el ensayo clínico alemán CoCStom que trata de acotar el momento óptimo para el cierre del estoma de protección en el contexto de la quimioterapia adyuvante, y del que aún no se han presentado resultados [78].

En los estudios revisados es llamativo el alto porcentaje de pacientes que presenta retrasos en la reconstrucción del tránsito.

En pacientes con cáncer de recto en estadios II-IV el intervalo está condicionado por la necesidad de tratamiento adyuvante, determinando que se deba retrasar el cierre del estoma tras finalizar el mismo [68-69]. El estudio multicéntrico alemán sobre 120 pacientes con EP inicial tras RAB estima un tiempo medio hasta el cierre de 5 meses (rango: 17 días a 18 meses). Un 3.4\% (4 pacientes) se reconstruyeron en los primeros 30 días. La quimioterapia adyuvante supuso un incremento significativo del tiempo medio hasta cierre del estoma (5.6 vs 3.4 meses; $p$ 0.0001) [63]. 


\section{Archivos de Coloproctología}

Archivos de Coloproctología, 2019; 3(3):05-47 DOI: https://doi.org/10.26754/ojs_arcol/arch_colo.201934001

estudio publicado por Waterland et al. en 2015 trata de definir además las razones del retraso en el cierre del estoma. Este estudio presenta una tasa de cierre del $75 \%$, con una mediana de tiempo hasta el cierre de 6 meses (rango 1-42 meses). En 63 pacientes que presentan retraso del cierre las causas fueron: quimioterapia adyuvante 35\% (22), patología médica $22 \%$ (14), fuga anastomótica 14\% (9), y otras 7\% (4). Se presentaron complicaciones en un $26 \%$ (33 pacientes) tras el cierre del estoma, y el análisis univariante mostró una influencia del cierre tardío del estoma con un incremento de la tasa de complicaciones y estancia hospitalaria [70].

En un estudio reciente realizado en Reino Unido sobre 203 pacientes intervenidos con RAB, sobre los 140 pacientes con EP realizado en la primera cirugía se observó que el $55.7 \%$ (78) seguían siendo portadores de estoma un año después de la intervención inicial. Según los autores existen diversos factores relacionados con esta alta tasa de permanencia de los EP, entre los cuales destacan estenosis de la anastomosis (14.1\%), situación clínica desfavorable (14.1\%), complicaciones postoperatorias de la cirugía original (12.8\%), recurrencia local o a distancia de la enfermedad neoplásica (18\%), complicaciones de la quimioterapia adyuvante (3.9\%), sospechas de anomalías detectadas en las imágenes radiológicas que requieren completar estudio (5.1\%), o los retrasos en la lista de espera (25.6\%) [21].

Hay que tener en cuenta que un porcentaje no desdeñable de pacientes no se llegarán a reconstruir. Según las cifras publicadas por los estudios 1 de cada 46 estomas inicialmente temporales no será cerrado $[5,70]$.

El estudio de Pan et al. recoge los datos de 296 pacientes con RAB con EP. Al final del seguimiento un $17.2 \%$ de pacientes seguían portando el estoma. El tiempo medio del intervalo desde la creación del estoma hasta la reconstrucción se mostraba variable con un rango entre 14 y 865 días. El análisis multivariante refleja que la enfermedad metastásica (OR 0.179, $\mathrm{p}<0.001$ ), un "Charlson 


\section{Archivos de Coloproctología}

Archivos de Coloproctología, 2019; 3(3):05-47 DOI: https://doi.org/10.26754/ojs_arcol/arch_colo.201934001

Comorbidity Index score" $>1$ (OR 0.268, p < 0.01) y las complicaciones tras la primera cirugía (OR 0.391, p 0.013) resultan factores de riesgo independientes para que no se realice el cierre del estoma [5].

El estudio publicado por Haksal et al. también tiene como objetivo definir factores de riesgo de no reconstrucción de la ileostomía de protección tras RAB. Analiza los resultados de 160 pacientes con RAB e ileostomía de protección, de los cuales 24 (15\%) no fueron reconstruidos. Las causas principales de la ausencia de reconstrucción fueron la estenosis de la anastomosis 54.2\%(13), rechazo del paciente a cierre del estoma 16.7\%(4), problemas urinarios (incontinencia urinaria, nefrostomía percutánea y fístula de ureteroneocistostomía) 12.5\%(3), comorbilidades y mala situación clínica 8.3\%(2), fístula anastomótica 8.3\%(2). El resultado del análisis multivariante señala como factores de riesgo independientes para la permanencia del estoma: el sexo masculino (OR 7.8, IC 95\% 1.34-45.67) y la resección multiorgánica (OR 6.71, IC 95\% 1.24-36.2). En este estudio el análisis de Kaplan Meier reveló una supervivencia a 5 años similar en los pacientes cuyos estomas fueron cerrados y en los que no $(80.8 \%$ vs $72.2 \%, p$ 0.143) [71]. Una tasa similar de falta de cierre es la que encuentra un estudio retrospectivo de 2017 sobre 520 pacientes con EP tras RAB, en el cual un $11.9 \%$ (62) de pacientes no fueron reconstruidos [72].

El estudio de Gustafsson et al. tiene por objetivo identificar factores asociados al "timing" de cierre del EP y para ello analiza los datos de 3564 pacientes. De ellos un $82.9 \%$ (2954) fue reconstruido con una mediana de intervalo de tiempo hasta el cierre de 191 días (rango 5-458 días). El cierre se produjo dentro de los estándares considerados ( 9 meses en pacientes sin adyuvancia o 12 meses en pacientes con quimioterapia adyuvante) en el $82.9 \%$. Los factores que se relacionaron con un mayor intervalo de tiempo hasta el cierre del EP fueron: complicaciones postoperatorias (OR 0.67, IC 95\% 0.50-0.81) y estadios avanzados del cáncer de recto (III y IV) (OR 0.19, IC 95\% 0.13-0.27). El retraso 


\section{Archivos de Coloproctología}

Archivos de Coloproctología, 2019; 3(3):05-47

DOI: https://doi.org/10.26754/ojs_arcol/arch_colo.201934001

del cierre y la ausencia de cierre se relacionan con un mayor ASA (ASA 1: OR 1.26, IC 95\% 1.03-1.53; ASA 3-4: OR 0.77, IC 95\% 0.61-0.96). En el estudio multivariante, los factores que muestran asociación con el tiempo hasta el cierre del estoma son: complicaciones de la cirugía inicial (HR 0.67, IC 95\% 0.62-0.73) y la quimioterapia adyuvante (HR 0.63, IC 95\% 0.57-0.69). Los factores asociados con una disminución de la probabilidad de cierre son un estadio tumoral avanzado (Estadio III: HR 0.74, IC 95\% 0.66-0.83, p < 0.001, Estadio IV: HR 0.38, IC 95\% 0.32-0.46, p < 0.001) y un ASA elevado (ASA3-4 HR 0.80, IC $95 \% 0.71-0.90, p<0.001)$. Los autores concluyen que en los pacientes en riesgo de no producirse reconstrucción se podría considerar el estoma permanente [73]

\section{Morbilidad del cierre del estoma de protección.}

La reconstrucción del EP presenta una morbilidad de hasta el 45.9\%. Algunas publicaciones reflejan una mortalidad de hasta el $6 \%$ y una tasa de reintervenciones del 7\% [50,68-69,74].

La revisión de Chow et al. que recoge los datos de 46 estudios, con un total de 6107 pacientes, comunica una morbilidad del cierre de ileostomía de protección del $17.3 \%$ y una mortalidad de $0.4 \%$ [65]. El estudio del grupo de Vigo publicado en Cirugía Española hace 3 años presenta un índice de complicaciones tras el cierre de la ileostomía de protección de $24 \%$, con un $5 \%$ de reintervenciones [50]. En la revisión de Chow et al. las complicaciones más frecuentes fueron la obstrucción intestinal y la infección de pared abdominal. En el estudio de Pérez Domínguez et al. las complicaciones mas observadas fueron la infección de herida y el íleo prolongado postoperatorio [65,50].

Entre los artículos más recientes encontramos dos estudios que analizan los posibles factores de riesgo de presentar complicaciones tras la reconstrucción.

El estudio de Nakamura et al. de 2017 analiza los resultados en 240 pacientes con estomas de protección en contexto de cirugía rectal (RAB y resecciones interesfinterianas). El análisis univariante y multivariante concluye con 3 factores 


\section{Archivos de Coloproctología}

Archivos de Coloproctología, 2019; 3(3):05-47

DOI: https://doi.org/10.26754/ojs_arcol/arch_colo.201934001

de riesgo independientes relacionados con el riesgo de complicaciones tras la cirugía de reconstrucción: la edad (OR 3.42, p 0.0025), el intervalo entre la cirugía inicial y el cierre del estoma (OR 3.48, p 0.0039), y el tiempo quirúrgico (OR 2.52, p 0.026) [75].

Entre las complicaciones del cierre del EP está incluida la posibilidad de ser necesario un nuevo estoma. El estudio de Song $O$ et al. tiene por objetivo identificar los factores de riesgo de re-confección de estoma tras el cierre de un EP analizando los resultados de 520 pacientes con ileostomía tras RAB o resección interesfinteriana por cáncer de recto. De ellos un 9.8\% (45) volvieron a ser ostomizados tras el cierre del EP. Las causas de re-confección del estoma fueron: complicaciones de la anastomosis 57.8\% (26), recurrencia local $33.3 \%$ (15), disfunción del esfínter anal 6.7\% (3). El análisis multivariante destacó como factores de riesgo independientes para la re-confección de estoma: la fuga anastomótica (OR 4.25, IC 95\% 1.814-9.993), la radioterapia postoperatoria (OR 3.947, IC 95\% 1.624-9.594) y la resección interesfintérica (OR 3.293, IC 95\% 1.462-7.417) [72].

Algunos autores refieren un índice de complicaciones significativamente más elevado en los pacientes que han recibido neoadyuvancia. En un análisis multivariante sobre 5.401 pacientes esta relación deja de ser significativa al incluir otros factores [76-77] y en otros trabajos no se ha observado esta relación $[50,69,74]$.

Los índices de morbilidad, la afectación de la calidad de vida y esfera psicosocial, la necesidad de una reintervención para reconstrucción del tránsito con una morbimortalidad asociada, la posibilidad de no reconstrucción del estoma, la posible interferencia en el tratamiento adyuvante, y los aún no conocidos efectos sobre la supervivencia a largo plazo e historia oncológica, unidos a los costes sanitarios que suponen, hacen que el empleo del estoma de protección siga siendo controvertido y que en los últimos años hayan surgido nuevas opciones 


\section{Archivos de Coloproctología}

Archivos de Coloproctología, 2019; 3(3):05-47

DOI: https://doi.org/10.26754/ojs_arcol/arch_colo.201934001

de manejo y nuevas técnicas para realizar la derivación fecal buscando reducir las complicaciones asociadas. A continuación haremos un repaso de las últimas controversias en cirugía colorrectal en cuanto a alternativas en el manejo del estoma de protección (cierre precoz) y otras formas de derivación fecal.

\section{Cierre precoz.}

En los últimos años ha surgido una corriente de autores que promueven el cierre precoz (CP) del EP. Se define como CP del estoma el que se realiza en las 2 semanas posteriores a la intervención inicial. A pesar de los resultados prometedores de algunos estudios, aún no existe suficiente evidencia para estandarizar su empleo.

Uno de los primeros artículos en estudiar el CP es un ensayo multicéntrico prospectivo aleatorizado con 186 pacientes que concluye que el cierre del EP en el $8^{\circ}$ día postoperatorio es seguro, sin un aumento de la tasa de complicaciones peroperatorias en su serie. A pesar de una mayor tasa de complicaciones de la herida (incluido el lugar del estoma previo), el cierre precoz se asoció con menos complicaciones médicas (CP $5 \%$ vs no CP 15\%, p 0.021) y menor tasas de obstrucción intestinal (CP $3 \%$ vs no CP 16\%, p 0.002) [79]. En la revisión sistemática publicada en 2015 por Robertson et al. no se llegó a ninguna conclusión debido a la heterogeneidad entre los estudios publicados [80].

El estudio aleatorizado de Lasithiotakis et al. tiene por objetivo evaluar los resultados del cierre precoz del EP tras RAB. EI CP fue significativamente superior en cuanto a la facilidad del cierre de la pared abdominal y de la reversión, duración de la intervención (mediana (RIC) 20 (13) vs 40 (9) min, $\mathrm{p}<0.01)$ y los costes derivados de la atención del estoma (mediana (RIC) $27(9)$ vs 311 (108) £, p <0.01). La estancia hospitalaria resultó similar en ambos grupos. No se presentaron complicaciones mayores en ninguno de los grupos. Este estudio concluye que en paciente seleccionados el cierre precoz del estoma 
Archivos de Coloproctología, 2019; 3(3):05-47

DOI: https://doi.org/10.26754/ojs_arcol/arch_colo.201934001

es factible, más fácil técnicamente, con un tiempo operatorio inferior, y además puede suponer una disminución de los costes [81].

En el metaanálisis publicado en 2017 por Farag et al. sobre 4 EA, que recoge los datos de 446 pacientes con EP tras RAB. 176 pacientes fueron sometidos a cierre precoz y 270 fueron reconstruidos de forma tardía. Los resultados no encuentran diferencias significativas entre los grupos de cierre precoz y tardío en cuanto a riesgo de fuga anastomótica (RR 0.37, IC 95\% 0.1-1.45, p 0.15), estenosis de la anastomosis (RR 4.79, IC 95\% 0.23-98.47, p 0.31) y complicaciones postoperatorias (RR 0.75, IC 95\% 0.48-1.16, p 0.19). Tampoco se observan diferencias significativas en cuanto al tiempo quirúrgico y estancia hospitalaria [82].

El reciente metaanálisis publicado por Menahem et al. que recoge los datos de 6 estudios, analizando los resultados sobre 570 pacientes, de los cuales 252 han sido sometidos a CP del estoma. En el estudio no se encuentran diferencias significativas en cuanto a la tasa de morbilidad entre los grupos CP / No CP (OR 0.63, IC 95\% 0.22-1.78, p 0.38). A pesar de una mayor tasa de infección de herida en la localización previa del estoma (OR 3.83, IC 95\% 2.14-6.86, p 0.00001 ), no se observan diferencias significativas en cuanto a la tasa de FA (OR 0.63, IC 95\% 0.22- 1.78, p 0.38). Las complicaciones relacionadas con el estoma (OR 0.46, IC 95\%

0.24-0.86, p 0.02), y la tasa de obstrucción intestinal (OR 0.11, IC 95\% 0.06-0.2, $\mathrm{p}<0.00001$ ) se presentan significativamente menores en el grupo de CP [46].

El último estudio encontrado es un EA publicado por Klek et al. va un paso más allá sugiriendo incluir el CP en los protocolos ERAS (Enhanced Recovery After Surgery) en cirugía de recto. El análisis comparativo entre CP y cierre tardío no encontró diferencias significativas en cuanto a tiempo quirúrgico, estancia hospitalaria e íleo postoperatorio. Si se observa diferencia estadísticamente significativa en cuanto al inicio del tratamiento adyuvante $(38.7 \pm 5.7$ vs $33.2 \pm 5.8$ 
Archivos de Coloproctología, 2019; 3(3):05-47

DOI: https://doi.org/10.26754/ojs_arcol/arch_colo.201934001

días, $\mathrm{p}<0.01$ ), reducción del tiempo con estoma (17.2 vs 299 días) y disminución de gastos sanitarios (43.68 vs 698.42) [83].

A falta de nuevos estudios que aporten mayor evidencia, los resultados recogidos en la literatura parecen apuntar que el cierre precoz del estoma de protección tras $\mathrm{RAB}$ es efectivo y seguro en pacientes seleccionados, sin incremento de la morbilidad postoperatoria. La aplicación de esta medida es recomendable de cara a reducir las complicaciones de los estomas, disminuir los gastos, y garantizar una administración precoz del tratamiento adyuvante lleostomía umbilical.

El ombligo, un orificio embrionario fisiológico, se utiliza cada vez más como vía de acceso para la cirugía laparoscópica de incisión única (SILS - Single incisión laparoscopic surgery). Algunos autores han propuesto, como parte de algunos de estos procedimientos, confeccionar un estoma de protección temporal a través del ombligo. Lejos de lo que pueda parecer, las ileostomías umbilical es están descritas en la literatura, empleadas desde los años 80-90 en cirugía pediátrica. [84].

Las ventajas teóricas del emplazamiento umbilical de la ileostomía pueden ser: una mayor preservación de la pared abdominal y mejores resultados estéticos. Uno de los primeros estudios en aplicar esta técnica a las ileostomías de protección en cirugía colorrectal es el publicado por Eto K. et al. en el que se incluyen 20 pacientes sometidos a RAB con EP, 10 de los cuales fueron realizados según la técnica tradicional, y los otros 10 a nivel umbilical. Los resultados no encuentran diferencias entre los grupos en cuanto a tiempo quirúrgico medio en la cirugía inicial (248 min vs $344 \mathrm{~min}$ ) y la estancia hospitalaria mediana después de la cirugía inicial (13 días vs 16 días). Las tasas de complicaciones después de la cirugía inicial fueron similares en ambos grupos, así como el tiempo quirúrgico promedio en el cierre del estoma (99 min vs $102 \mathrm{~min}$ ) y la estancia hospitalaria mediana tras el cierre del estoma (8 días 


\section{Archivos de Coloproctología}

Archivos de Coloproctología, 2019; 3(3):05-47

DOI: https://doi.org/10.26754/ojs_arcol/arch_colo.201934001

en ambos grupos). Las complicaciones tras el cierre del estoma fueron similares en ambos grupos [85].

El estudio publicado por Mushava et al. es una serie de casos prospectiva en la que se incluyeron pacientes con indicación de ileostomía de protección en cirugía colorrectal urgente y electiva. Se realizaron 12 estomas umbilicales, con un tiempo medio de duración hasta el cierre de 113 días. La morbilidad global más frecuente fue la relacionada con la piel periestomal. Según los resultados 3 pacientes presentaron estoma invertido con excelente resultado estético [86]. El estudio de Miyo $\mathrm{M}$ et al. recopila los datos de 83 pacientes intervenidos por neoplasia de recto con RAB con ileostomía de protección. De ellos 53 fueron ostomizados con ileostomía convencional, y 30 con ileostomía umbilical. Ambos grupos presentan una tasa similar de complicaciones postoperatorias $(26.7 \%$ vs $32.1 \%$, p 0.804) y relacionadas con el estoma (3.3\% vs 3.8\%, p 1.000) [87].

El último estudio publicado (2018) evalúa los resultados de forma retrospectiva en 234 pacientes tras la implantación del SILS en la cirugía colorrectal con indicaciones benignas y malignas. De los pacientes ostomizados (74 / 31.6\%) (ileostomías (41) y colostomías (33)), se realizaron estomas umbilicales en el $27 \%$ (20 pacientes). De ellos, la morbilidad relacionada con el estoma se produjo en el 5\% (1 paciente con vólvulo de torsión ileal). La mediana de seguimiento desde la confección del estoma fue de 30 meses (rango 12-59 meses). La calidad de vida y la funcionalidad del estoma fueron adecuados según lo estimado por el estomatoterapeuta. Después de la reversión del estoma, dos pacientes fueron reintervenidos por hernia incisional (10\%) [88].

\section{lleostomía transcecal.}

Entre las alternativas al estoma convencional que encontramos en la literatura, está la ileostomía transcecal. Consiste en la colocación de un catéter insertado a través del ciego y de la válvula ileocecal, con el extremo distal en íleon terminal. 


\section{Archivos de Coloproctología}

Archivos de Coloproctología, 2019; 3(3):05-47

DOI: https://doi.org/10.26754/ojs_arcol/arch_colo.201934001

Esta técnica puede tener como ventajas potenciales menores complicaciones y además evita una reintervención para el cierre del estoma.

El trabajo de Monzón-Abad et al. incluye 18 pacientes con neoplasias de recto medio-inferior con RAB o RAUB y ETM en los que se colocó un catéter de ileostomía transcecal. Dos pacientes desarrollaron celulitis periférica a la salida del catéter (11.1\%). En ambos casos la curación se logró en los primeros 30 días tras la cirugía. Un paciente presentó una FA (5.5\%) tras retirar el catéter. La estancia hospitalaria media fue de 10 días (rango 8-13 días) [89].

\section{Tubo de ileostomía con auto-cierre (TIAC)}

Uno de los primeros artículos encontrado en la literatura aplica esta técnica de forma preliminar en 3 pacientes con proctocolectomia total con anastomosis Pouch-anal. En este trabajo se describe la técnica con cierre del margen eferente intestinal con una grapadora, aproximadamente a $10 \mathrm{~cm}$ de la inserción del tubo. El tubo empleado es un tubo endotraqueal del nํㄱ, que se introduce a través de la pared abdominal a nivel de FID y se inserta en el intestino delgado a través del margen aferente [Figura 1].

Según los resultados en esta aplicación preliminar los autores concluyen que el TIAC proporciona la misma protección que un estoma convencional, puede permitir un cierre espontáneo del estoma tras la extracción del tubo evitando la segunda cirugía y genera una incisión y herida de menores dimensiones, lo que implica mayor comodidad en los cuidados postoperatorios [90].

El primer estudio comparativo presenta los resultados de 95 pacientes sometidos a RAB por cáncer de recto. 54 fueron tratados con dispositivo TIAC (56\%) y 41 (44\%) con ileostomía de protección convencional. Los resultados no encontraron diferencias entre las tasas de FA entre los grupos (5.6\% y $4.9 \%$ ). En el grupo TIAC, se produjo la repermeabilización espontánea del extremo eferente en un 


\section{Archivos de Coloproctología}

Archivos de Coloproctología, 2019; 3(3):05-47 DOI: https://doi.org/10.26754/ojs_arcol/arch_colo.201934001

tiempo medio de 27.9 días (14-70). El tubo se retiró a los 33 días de media (2075) y el estoma restante se cerró a los 12.3 días (6-30) [91].

Un nuevo estudio comparativo entre la técnica TIAC y el estoma convencional presenta unos resultados alentadores. No se observan diferencias en cuanto a la tasa de fugas anastomóticas (ileostomía 8.1\% (12/149) vS TIAC 8.3\% (12/145), p1) ni en la tasa de reintervención (ileostomía 3\% (4/149) vs TIAC 3.4\% (5/145), p 0.75). La estancia media hospitalaria fue mayor en el grupo con ileostomía convencional (ileostomía 17.1 días vs TIAC 8.6 días, $p<0.01$ ). Durante el tiempo de seguimiento del estudio 13 pacientes con ileostomía permanecieron con estoma, mientras que solo 2 pacientes del grupo TIAC seguían portando estoma $(p<0.01)[92]$.

El estudio de Chen et al. compara la técnica con TIAC y el estoma convencional en 41 pacientes. Los resultados obtenidos con el tubo de ileostomía revelan beneficio sobre la duración de la estancia postoperatoria (7 (RIC 7-9) vs 13 (RIC 13-15.25), $\mathrm{p}<0.001$ ), los costes sanitarios (6.5 (RIC 6.4-6.7) vs 11.35 (RIC 11.111.6), $\mathrm{p}<0.001$ ) y el intervalo hasta el cierre del estoma (34 (RIC 31-37) vs 95 (91-99), $p<0.001)$ con una diferencia estadísticamente significativa. Según los resultados expuestos no se objetivaron complicaciones asociadas al TIAC y no se identificaron diferencias significativas en las complicaciones más frecuentes, incluido el sangrado de la sutura, la fuga anastomótica, la estenosis anastomótica y la infección de herida entre los grupos [93].

La mayor evidencia al respecto del uso del tubo de ileostomía lo aporta el metaanálisis publicado en 2016 por Zong $Z$ et al. que recoge los resultados de 4 estudios, con un total de 642 pacientes. De ellos 332 habían sido tratados con TIAC y 310 con estoma convencional en asa. Los resultados en cuanto a incidencia de fugas anastomóticas y tasa de reintervenciones fueron 


\section{Archivos de Coloproctología}

Archivos de Coloproctología, 2019; 3(3):05-47 DOI: https://doi.org/10.26754/ojs_arcol/arch_colo.201934001

estadísticamente similares en los dos grupos. En comparación con el estoma de asa convencional, el TIAC presentó menos complicaciones relacionadas con el estoma (RR 0.2, IC 95\% 0.05- 0.5), menor tiempo quirúrgico (diferencia de medias ponderada $=-47.28$ minutos, IC del 95\%: -74.68 a -19.88) y menor estancia hospitalaria (diferencia de medias ponderada = -5.22 días, IC del 95\%: -10.32 a -0.13, respectivamente). El tiempo hasta el cierre del estoma fue significativamente menor en el grupo con tubo (diferencia de medias ponderada -114 días, IC 95\% -148.38 a -80.77) [94].

\section{lleostomía virtual o ileostomía fantasma.}

En 2007 el trabajo de Sacchi et al. describe la "ileostomía virtual" (IV), una técnica que surge como alternativa al estoma convencional para proteger las anastomosis de alto riesgo [95].

\section{Técnica.}

Una vez finalizada la fase reconstructiva de la anastomosis se pasa un "Vessel Loop" referenciando el íleon terminal, a $10 \mathrm{~cm}$ aproximadamente de la válvula ileocecal, a través de una ventana en el mesenterio, adyacente a la pared ileal, evitando lesionar el mismo. Se coloca una sutura para marcar el segmento proximal y se exterioriza el "Vessel Loop" a través de la pared abdominal sin tensión y se asegura a la piel sobre una varilla. Si no hay indicios de dehiscencia anastomótica se retirará el vessel Loop a los 8-12 días de la cirugía. Si hubiese signos de complicación anastomótica se realizará una ileostomía realizando una incisión adyacente al vessel loop, sin necesidad de realizar una nueva laparotomía [96] [Figura 2].

Esta técnica, según los estudios publicados que avalan su efectividad, no disminuye la tasa de FA, pero sí disminuye el número de ileostomías temporales 
Archivos de Coloproctología, 2019; 3(3):05-47

DOI: https://doi.org/10.26754/ojs_arcol/arch_colo.201934001

hasta un $80-90 \%$, sin suponer un aumento de la morbilidad postoperatoria. Además los autores aseguran que es una técnica segura, fácil de realizar y económica. Su uso apropiado requiere una sincronización precisa: el diagnóstico precoz de la dehiscencia de anastomosis mediante un drenaje perianastomótico o la obtención de imágenes (radiológicas o rectoscopia) y la exteriorización del asa ileal lo antes posible una vez se identifican los signos/síntomas sugestivos de dehiscencia [97].

El primer estudio, realizado por Sacchi et al. describe los resultados tras la aplicación en 107 pacientes intervenidos por cáncer de recto con RAB. En los 14 pacientes que presentaron clínica sugestiva de FA (13\%) se utilizó la "ileostomía virtual" para realizar una ileostomía en asa clásica. Este procedimiento redujo en más del $80 \%$ el número de ileostomías inicialmente planificado [95].

El estudio de Marrosu A. et al presenta resultados preliminares tras la aplicación de esta técnica en 49 pacientes tras RAB por cáncer de recto. En 27 de ellos se decidió hacer una ileostomía, y en 22 una IV. Se observó una tasa similar de FA en ambos grupos (5 pacientes (18.5\%) en grupo ileostomía vs 4 pacientes (18.2\%) en grupo IV). En el grupo con IV los 18 pacientes que no presentaron signos de FA, fueron liberados del material de referencia (Vessel Loop) entre los 9-11 días sin complicaciones asociadas. Los 4 pacientes que presentaron signos clínicos de FA fueron convertidos rápidamente a ileostomía en asa usando el Vessel Loop de referencia como guía. Tras la confección de la ileostomía en asa se produjo un descenso de los signos y síntomas de la A. No se objetivaron diferencias significativas entre los grupos [97].

El estudio de Mori et al. presenta los resultados obtenidos con la aplicación de la IV en 168 pacientes. De ellos se presentaron síntomas sugestivos de dehiscencia en 20 (11.96\%), entre el $4^{\circ}$ y $12^{\circ}$ días de postoperatorio. De ellos, en 13 pacientes (65\%) se realizó una ileostomía y no fue necesaria una reintervención urgente, en 5 casos (25\%) la complicación se resolvió con 


\section{Archivos de Coloproctología}

Archivos de Coloproctología, 2019; 3(3):05-47 DOI: https://doi.org/10.26754/ojs_arcol/arch_colo.201934001

tratamiento conservador (no se realizó ileostomía ni reintervención) y sólo en 2 casos $(10 \%)$ se produjo una peritonitis generalizada requiriendo una reintervención con lavado y confección de colostomía. 153 pacientes (91.2\%) no precisaron realización de estoma. Las conclusiones de este estudio destacan al indicar la posibilidad de crear una ileostomía en asa de forma selectiva, sin necesidad de hacer una laparotomía. Los autores matizan que debe indicarse la IV en pacientes de bajo riesgo de FA [96].

El estudio de Mari FS et al. es un ensayo aleatorizado que compara los resultados obtenidos con la aplicación de IV y no aplicación de estoma en 107 pacientes. En el grupo de IV, con 55 pacientes, se objetivaron 3 FA (5.5\%), y en el grupo de No estoma, con 52 pacientes, se produjeron 4 FA (76.9\%). En el grupo de IV los pacientes con dehiscencia anastomótica presentaron menor severidad clínica, menor estancia hospitalaria, y ninguno precisó realizar laparotomía. La conclusión final de los autores destaca la posibilidad, gracias a la IV, de reducir el número de estomas realizados [98].

El reciente de estudio de Ambe PC et al. aplica la ileostomía virtual tras la proctocolectomía por poliposis adenomatosa familiar en 8 pacientes. La IV se liberó entre los días $7^{\circ}$ y $9^{\circ}$ de postoperatorio. No se registró ninguna dehiscencia de anastomosis. En este caso se considera la IV como una buena alternativa para evitar la ileostomía temporal en pacientes que no lo necesitan [99].

\section{Dispositivo de derivación fecal.}

Recientemente un estudio Coreano presenta como alternativa a la derivación fecal para prevenir la sepsis asociada a la dehiscencia de anastomosis la aplicación de un dispositivo de derivación fecal, anteriormente utilizado de forma experimental en animales con resultados satisfactorios. El Sistema DDF consta 


\section{Archivos de Coloproctología}

Archivos de Coloproctología, 2019; 3(3):05-47 DOI: https://doi.org/10.26754/ojs_arcol/arch_colo.201934001

de 2 dispositivos principales: EI DDF y la banda para su fijación. EI DDF [Figura 3] es un dispositivo tubular de silicona, compuesto por una cabeza tubular sólida y una parte tubular delgada. La cabeza tiene 2 globos exteriores y un globo interior. Los globos exteriores sirven para fijar el DDF en el colon, y el globo interior se usa para detener el flujo en el tubo. Paralelo a los catéteres para los globos, hay un canal para la infusión de suero. La parte tubular delgada o "cola" es lo suficientemente larga para sobresalir por el ano.

La banda es de ac. Poli-láctico-glicólico (PGLA), un material absorbible con una vida media de 6 semanas [Figura 4]. La banda extraluminal abraza la zona estrecha entre los dos balones para fijar el DDF en la posición deseada dentro del colon. Para poder aplicar la banda de forma segura se acompaña de un instrumento medidor de presión.

Procedimiento de colocación del DDF. Tras la extracción de la pieza se introduce el dispositivo medidor en el mesocolon, para calcular la longitud adecuada de la banda. Se introduce la banda, que se fija en el mesocolon a la altura calculada con dos puntos de seda $2 / 0$ que se dejan sueltos. Tras la realización de la anastomosis se inserta el DDF a través del ano hasta la zona marcada con la banda, con el balón distal desinflado y el proximal inflado. Una vez inflado el balón externo se anudan los 2 puntos de sutura [Figura 5].

En el manejo postoperatorio es importante la irrigación con agua dos veces al día desde el día después al inicio de la dieta líquida oral. El tiempo medio de permanencia del dispositivo es de 3 semanas, que se prolonga si existe dehiscencia anastomótica.

En el estudio piloto se realiza un TC abdominal a la semana tras la cirugía para descartar una FA asintomática, y 3 semanas tras la cirugía se realiza un estudio radiológico con contraste hidrosoluble. Si no hay evidencia de FA, se retira el DDF. Si hay evidencia de FA (sinus) se recomienda mantener el DDF otras 1-3 semanas. La duración máxima del DDF es 6 semanas de acuerdo con la vida 


\section{Archivos de Coloproctología}

Archivos de Coloproctología, 2019; 3(3):05-47 DOI: https://doi.org/10.26754/ojs_arcol/arch_colo.201934001

media del material de la banda. En el estudio además se realizó sigmoidoscopia tras la retirada para evaluar la anastomosis y descartar lesiones producidas por la banda y el DDF.

El estudio expone los resultados de la aplicación del DDF en 31 pacientes. 10 pacientes (32\%) presentaron FA detectada en el TC abdominal realizado a la semana tras la cirugía. Solo uno de ellos presentó una complicación mayor, Clavien Dindo grado IIla, por una colección perianastomótica que se trató con drenaje percutáneo. El enema opaco a las 3 semanas reveló en 5 casos la persistencia de sinus (16\%). El tratamiento conservador en estos casos, incluyendo la permanencia del DDF 1-3 semanas más fue suficiente para mantener la anastomosis. En 3 casos se objetivaron erosiones en la pared del colon en relación con el DDF. Los autores argumentan las bondades del dispositivo que en la serie fue efectivo para prevenir la septicemia y evitar una reintervención y un estoma temporal [100].

\section{CONCLUSIÓN}

Los estomas de protección son una medida eficaz para controlar la morbilidad de la dehiscencia anastomótica tras la RAB por cáncer de recto. A pesar de algunos estudios con resultados controvertidos, los últimos EA y metaanálisis demuestran que el estoma de protección tiene un efecto protector sobre la tasa de fuga anastomótica y reintervenciones urgentes. Hasta ahora ningún estudio con suficiente nivel de evidencia incluye datos en referencia a la influencia sobre la supervivencia a largo plazo y calidad de vida. El estoma de protección afecta a la calidad de vida del paciente y tiene una morbilidad específica asociada. Además precisa una nueva intervención para su reconstrucción, la cual tiene una morbimortalidad añadida. Estas complicaciones pueden interferir en el desarrollo 


\section{Archivos de Coloproctología}

Archivos de Coloproctología, 2019; 3(3):05-47

DOI: https://doi.org/10.26754/ojs_arcol/arch_colo.201934001

del tratamiento adyuvante, aunque aún no hay datos suficientes para aclarar este efecto. Dentro de las complicaciones del estoma está la permanencia del mismo, la cual se puede producir hasta en uno de cada 4 pacientes ostomizados con intención transitoria.

Estas particularidades hacen necesario una selección adecuada de los pacientes que reciben estoma de protección tras $\mathrm{RAB}$ por cáncer de recto.

En los últimos años ha surgido una corriente que boga por el cierre precoz (en los primeros 15 días) sin un incremento de la morbilidad, pero aún no hay suficiente evidencia para estandarizar su uso. Asimismo, diversos autores han publicado terapias alternativas al estoma en asa clásico, que es importante conocer, pero aún están en proceso de estudio y validación.

\section{BIBLIOGRAFÍA}

1. Hanna MH, Vinci A, Pigazzi A. Diverting ileostomy in colorectal surgery: When is it necessary? Langenbeks Arch Surg (2015) 400:145-152.

2. Armendáriz-Rubio $\mathrm{P}$, de Miguel Velasco $\mathrm{M}$, Ortiz Hurtado $\mathrm{H}$, et al. Comparación de colostomías e ileostomías como estomas derivativos tras resección anterior baja. Cir Esp. (2007) 81(3):115-20.

3. Ptok H, Marusch F, Meyer F, et al. Impact of anastomotic leakage on oncological outcome after rectal cancer resection. Br J Surg. (2007) 94(12):154854.

4. Law WL, Choi HK, Lee YM, et al. Anastomotic leakage is associated with poor long-term outcome in patients after curative colorectal resection for malignancy. J Gastrointest Surg (2007) 11(1):8-15.

5. Pan HD, Peng YF, Wang $L$, et al. Risk factors por nonclosure of a temporary defunctioning ileostomy following anterior resection of rectal cancer. Dis Colon Rectum. (2016) 59(2):94- 100. 


\section{Archivos de Coloproctología}

Archivos de Coloproctología, 2019; 3(3):05-47 DOI: https://doi.org/10.26754/ojs_arcol/arch_colo.201934001

6. Kang CY, Halabi WJ, Chaudhry OO, et al. Risk factos for anastomotic leakage after anterior resection for rectal cancer. JAMA Surg. 2013;148(1):6571.

7. Nisar PJ, Lavery IC, Kiran RP. Influence of neoadjuvant radiotherapy on anastomotic leak after restorative rsecion for rectal cancer. J Gastrointest Surg (2012) 16(9):1750-1757.

8. Sebag-Montefiore D, Stephens RJ, Steele R, et al. Preoperative radiotherapy versus selective postoperative chemoradiotherapy in patients with rectal cancer (MRC CR07 and NCIC-CTG C016): a multicentre, randomized trial. Lancet (2009) 373(9666):811-820.

9. Bertelsen $\mathrm{CA}$, Andreasen $\mathrm{AH}$, Jorgensen, et al. Anastomotic leakage after anterior resection for rectal cancer: risk factors. Color Dis (2010) 12(1):37-43.

10. Beard JD, Nicholson ML, Sayers RD, et al. Intraoperative air testing of colorrectal anastomoses: a prospective, randomized trial. Br J Surg (1990) 77:1095-1097.

11. Ivanov D, Cvijanovic R, Gvozdenovic $L$ Intraoperative air testing of colorectal anastomoses. Srp Arh Celok Lek (2011) 139:333-338.

12. Lieto $E$, Orditura $M$, Castellano $P$, et al. Endoscopic intraoperative anastomotic testing may avoid early gastrointestinal anastomotic complications: a prospctive study. J Gastrointest Surg (2011) 15:145-152.

13. Shamiyeh A, Szabo K, Ulf Wayand W, et al. Intraoperative endoscopy for the assessment of circular stapled anastomosis in laparoscopic colon surgery. Surg Laparosc Endosc Percutan Tech (2012) 22:65-67.

14. Bell SW, Walker KG, Rickard MJ et al. Anastomotic leakage after curative anterior resection in a higher prevalence of local recurrence. Br J Surg (2003) 90(10):1261-1266. 


\section{Archivos de Coloproctología}

Archivos de Coloproctología, 2019; 3(3):05-47

DOI: https://doi.org/10.26754/ojs_arcol/arch_colo.201934001

15. Katoh H, Yamashita K, Wang G et al. Anastomotic leakage contributes to the risk for systemic recurrence in stage II colorectal cancer. J Gastrointest Surg (2011) 15(1):120-129.

16. Mlrnezami A, Mirnezami R, Chandrakumaran K, et al. Increased local recurrence and reduced survival from colorectal cancer following anastomotic leak: systematic review and meta- analysis. Ann Surg (2011) 253(5):890-899.

17. Yao H, An Y, Zhang Z. The application of defunctioning stomas after low anterior resection of rectal cancer. Surg Today. 2018 Nov 27. doi: 10.1007/s00595-018-1736-6.

18. Shiomi $\mathrm{A}$, Ito $\mathrm{M}$, Maeda $\mathrm{K}$, et al. Effects of a diverting stoma on symptomatic anastomotic leakage after low anterior resection for rectal cáncer: a propensity score matching analysis of 1014 consecutive patients. J Am Coll Surg. 2015;220(2):186-94.

19. Shiomi $A$, Ito $M$, Saito $N$, et al. Diverting stoma in rectal cáncer surgery. A retrospective study of 329 patients from Japanese cancer centers. Int J Colorectal Dis. $2011 ; 26(1): 79-87$.

20. Gastinger I, Marusch F, Steinert R, et al. Protective defunctioning stoma in low anterior resection for rectal carcinoma. Br J Surg. 2005;92(9):1137-42.

21. Emmanuel A, Chohda E, Lapa C, et al. Defunctioning stomas result in significantly more short- term complications following low anterior resection for rectal cáncer. World J Surg. 2018;42:3755-3764.

22. Healthcare qualiti improvement partnership 2017. National bowel cáncer audit: Annual Report 2017. https://www.hqip.org.uk/resource/national-bowelcancer-audit-annual-report-2017.

23. Snijders HS, Van den Broek CBM, Wouters MWJM et al. An increasing use of defunctioning stomas after low anterior resection for rectal cancer. Is this the way to go? Eur J Surg Oncol. 2013;39:715-720. 


\section{Archivos de Coloproctología}

Archivos de Coloproctología, 2019; 3(3):05-47

DOI: https://doi.org/10.26754/ojs_arcol/arch_colo.201934001

24. Rutegard $M$, Boström $P$, Haapamäki $M$, et al. Current use of diverting stoma in anterior resection for cancer: population-based cohort study of total and partial mesorectal excision. Int J Colorectal Dis. 2016;31:579-585.

25. Szczepkowski M, Banasiewicz T, Krokowicz P, et al. Polish consensus statement on the protective stoma. Polski przeglad chirurgiczny. 2014;86(8):391404.

26. The American Society of Colon and Rectal surgeons. Dis Colon Rectum. 2014;57(5):e75-358.

27. Mowschenson PM, Critchlow JF, Peppercorn MA. Ileoanal pouch operation. Long-term outcome with or whitout diverting ileostomy. Arch Surg. 2000;135:463-466.

28. Machado M, Hallbook O, Goldman S et al. Defunctioning stoma in low anterior resection with colonic pouch for rectal cancer: a comparison between two hospitals with a different policy. Dis Colon Rectum. 2002;45:940-945.

29. Wong NY, Eu KW. A defunctioning ileostomy does not prevent clinical anastomotic leak after a low anterior resection: a prospective, comparative study. Dis Colon Rectum. 2005;48:2076- 2079.

30. Ikeuchi $H$, Nakano $H$, Uchino $M$ et al. Safety of one stage restorative proctocolectomy for ulcerative colitis. DIs Colon Rectum. 2005;48:1550-1555.

31. Remzi FH, Fazio VW, Gorgun E et al. The outcome after restorative proctocolectomy with orwithout defunctioning ileostomy. Dis Colon Rectum. 2006;49:470-477.

32. Gu WL, Wu SW. Meta-analysis of defunctioning stoma in low anterior resection with local mesorectal excision for rectal cancer: evidence based on thirteen studies. World J Surg Oncol. 2015;13:9.

33. Mattiessen $\mathrm{P}$, Hallbook $\mathrm{O}$, Anderson $\mathrm{M}$ et al. Risk factors for anastomotic leakage after anterior resection of the rectum. Color Dis. 2004;6:462-469. 


\section{Archivos de Coloproctología}

Archivos de Coloproctología, 2019; 3(3):05-47 DOI: https://doi.org/10.26754/ojs_arcol/arch_colo.201934001

34. Marusch F, Koch A, Schmidt $U$ et al. Value of a protective stoma in low anterior resections for rectal cancer. Dis Colon Rectum. 2002;45:1164-1171.

35. Peeters KC, Tollenaar RA, Arijnen CA et al. Risk factors for anastomotic failure after total mesorectal excision of rectal cancer. Br J Surg. 2005;92:211216.

36. Nurkin S, Kakarla VR, Ruiz DE et al. The role of faecal diversion in low rectal cancer: a review of 1791 patients having rectal resection with anastomosis for cancer, with and without a proximal stoma. Color Dis. 2013;15(6):e309-e316. 37. Belkin N, Bordeianou LG, Shellito PC, et al. Morbidity associated with diverting loop ileostomies: Weighing diversion in rectosigmoid resection. Am Surg. 2017;83(7):786-792.

38. Matthiessen P, Hallbook O, Rutegard J et al. Defunctioning stoma reduces symptomatic anastomotic leakage after low anterior resection of the rectum for cancer: a randomized multicenter trial. Ann Surg 2007;246:207-214.

39. Chude GG, Rayate NV, Patris V, et al. Defunctioning loop ileostomy with low anterior resection for distal rectal cancer: should we make an ileostomy as routine procedure? A prospective randomized study. Hepatogastroenterology 2008;55(86-87):1562-1567.

40. Huser N, Michalski CW, Erkan M, et al. Systematic review and metaanalysis of the role of defunctioning stoma in low rectal cancer surgery. Ann Surg 2008;248(1):411-414.

41. Montedori A, Cirocchi R, Farinella E, et al. Coverin ileo- or colostomy in anterior resection for rectal carcinoma. Cochranne Database of Syst Rev 2010;(5).Art.No.: CD006878.

42. Chen J, Wang DR, Yu HF, Zhao ZK, Wang LH, LI YK. Defunctioning stoma in low anterior resection for rectal cancer: a meta-analysis of five recent studies. Hepatogastroenterology. 2012;59(118):1828-31. 


\section{Archivos de Coloproctología}

Archivos de Coloproctología, 2019; 3(3):05-47

DOI: https://doi.org/10.26754/ojs_arcol/arch_colo.201934001

43. Wu SW, Ma CC, Yang Y. Role of protective stoma in low anterior resection for rectal cancer: a meta-analysis. World J Gastroenterol 2014;20(47):1803118037.

44. Güenaga KF, Lustosa SAS, Saad SS, et al. lleostomy or colostomy for temporary dexompresion of colorrectal anastomosis. Cochrane Database of Syst Rev, Issue 1. 2007. Art No.: CD004647.

45. Shellito PC. Complications of abdominal stoma surgery. Dis Colon Rectum. 1998;41:1562- 1572.

46. Menahem B, Lubrano J, Vallois A, et al. Early Closure of defunctioning loop ileostomy: Is it beneficial for the patient? A meta-analysis. World J Surg. 2018;42(10):3171-3178.

47. Londono-Schimmer EE, Leong AP, Phillips RK. Life table analysis of stomal complications following colostomy. Dis Colon Rectum. 1994;37(9):916920.

48. Chen F, Stuart M. The morbidity of defunctioning stomata. Aust N Z J Surg. 1996;66(4):218- 221.

49. Porter JA, Salvati EP, Rubin RJ, et al.Complications of colostomies. Dis Colon Rectum. 1989;32(4):299-303.

50. Pérez-Domínguez L, García-Martínez MT, Cáceres-Alvarado Nieves, et al. Morbilidad y mortalidad de la ileostomía derivativa temporal en la cirugía por cáncer de recto. Cir Esp. 2014;92(9):604-608.

51. Shabbir J, Britton DC. Stoma complications: A literatura overview. Colorectal Dis. 2010;12:958-64.

52. Harris DA, Egbeare D, Jones S, et al. Complications and mortality following stoma formation. Ann R Coll Surg Engl. 2005;87(6):427-31.

53. Cottam J, Richards K, Hasted A, et al. Results of a nationwide prospective audit of stoma complications within 3 weeks of surgery. Color Dis. $2007 ; 9(9): 834-838$. 


\section{Archivos de Coloproctología}

Archivos de Coloproctología, 2019; 3(3):05-47

DOI: https://doi.org/10.26754/ojs_arcol/arch_colo.201934001

54. Neuman HB, Park J, Fuzesi S, et al. Rectal cancer patients' quality of life with a temporary stoma: shifting perspectives. Dis Colon Rectum. 2012;55(11):1117-1124.

55. O'Leary DP, Fide CJ, Foy C, et al. Quality of life after low anterior resection with total mesorectal excision and temporary loop ileostomy for rectal carcinoma. Br J Surg. 2001;88(9):1216-1220.

56. Macdonald A, Chung D, Fell S, et al. An assessment of surgeons' abilities to site colostomies accurately. Surgeon. 2003;1(6):347-349.

57. American Society of Colon and Rectal Surgeons Committee Members. ASCRS and WOCN joint position statement on the value of preoperative stoma marking for patients undergoing fecal ostomy surgery. Wound Ostomy Continence Nurs 2007;34(6):627-8.

58. PJ A, Bevan L, Macdonald L, et al. A prospective audit of stomas analysis of risk factors and complications and their management. Color Dis. 2003;5(1):49-52.

59. Marquis P,Marred A, Jamb on B. Quality of life in patients with stomas: the Monteux Study. Ostomy Wound Manage. 2003;49(2):48-55.

60. Baker ML, Williams RN, Nightingale JM. Causeas and management of a high-output stoma. Color Dis. 2011;13(2):191-7.

61. Klink CD, Lioupis K, Binnebösel $\mathrm{M}$ et al. Diversion stoma after colorectal surgery: loop colostomy or ileostomy? Int J Color Dis. 2011;26(4):431-436.

62. Nastro $\mathrm{P}$, Knowles $\mathrm{CH}$, McGrath $\mathrm{A}$, et al. Complications of intestinal stomas. Br J Surg. 2010; 97(12):1885-1889.

63. Herrle F, Sandra-Petrescu F, Weiss C, et al. Quality of life and timing of stoma closure in patients with rectal cáncer undergoing low anterior resection with diverting stoma: A multicenter longitudinal observational study. Dis Colon Rectum. 2016;59(4):281-90. 


\section{Archivos de Coloproctología}

Archivos de Coloproctología, 2019; 3(3):05-47

DOI: https://doi.org/10.26754/ojs_arcol/arch_colo.201934001

64. Danielsen AK, Burcharth J, Rosenberg J. Patient education has a positive effect in patients with a stoma: a systematic review. Colorectal Dis. 2013;15(6):e276-83.

65. Chow A, Tilney HS, Paraskeva $P$, et al. The morbidity surrounding reversal of defunctioning ileostomies. A systematic review of 48 studies including 6107 cases. Int J Colorectal Dis. 2009;24:711-23.

66. Perez RO, Habr-Gama A, Seid VE, et al. Loop ileostomy morbidity: Timing of closure matters. Dis Colon Rectum. 2006;48:1539-45.

67. El-Hussana A, Lauritsen M, Bülow S. Relative high incidence of complication after loop ileostomy reversal. Dan Med J. 2012;59:A517.

68. Flikier-Zelkowicz B, Codina-Cazador A, Farrés-Coll R, et al. Morbilidad y mortalidad en relación con el cierre de ileostomías derivativas en la cirugía del cáncer de recto. Cir Esp. 2008;84:16-9.

69. Courtier R, Parés D, Silva CA, et al. Resultados clínicos del cierre de ileostomías en asa en pacientes intervenidos de cáncer de recto. Efecto de la quimioterapia en el tiempo de espera. Cir Esp. 2010;88:308-13.

70. Waterland P, Goonetilleke K, Naumann DN, et al. Defunctioning ileostomy reversal rates and reasons for delayed reversal: Does delay impact on complications of ileostomy reversal? A study of 170 defunctioning ileostomies. J Clin Med Res. 2015;7(9):685-689.

71. Haksal M, Okkabaz N, Atici AE, et al. Fortune of temporary ileostomies in patients treated with laparoscopic low anterior resection for rectal cancer. Ann Surg Treat Res. 2017;92(1):35-41.

72. Song O, Kim KH, Lee SY, et al. Risk factors of stoma re-creation after closure of diverting ileostomy in patients with rectal cancer who underwent low anterior resection or intersphincteric resection with loop ileostomy. Ann Surg Treat Res. 2018;94(4):203-208. 


\section{Archivos de Coloproctología}

Archivos de Coloproctología, 2019; 3(3):05-47

DOI: https://doi.org/10.26754/ojs_arcol/arch_colo.201934001

73. Gustafsson CP, Gunnarsson U, Dahlstrand U, Lindforss U. Loopileostomy reversal—patient- related characteristics influencing time to closure. Int J Colorectal Dis. 2018;33:593-600.

74. Mengual-Ballester M, García-Martín JA, Pellicer-Franco E, et al. lleostomías de protección: complicaciones y mortalidad asociadas a su cierre. Rev Esp Enferm Dig. 2012;104:350-4.

75. Nakamura $T$, Sato $T$, Naito $M$, et al. Risk factors for complications after divertins ileostomy closure in patients who have undergone rectal cáncer surgery. Surg Today. 2017;47(10):1238-1242.

76. Thalheimer A, Bueter M, Kortuem M, et al. Morbidity of temporary loop ileostomy in patients with colorectal cancer. Dis Colon Rectum. 2006;49:10111017.

77. Sharma A, Deeb P, Rickles $S$, et al. Closure of defunctioning loop ileostomy is associated with considerable morbidity. Colorectal Dis. 2013;15:4517.

78. Sandra-Petrescu F, Herrle F, Hinke A, et al. CoCStom trial: study protocol for a randomized trial comparing completeness of adjuvant chemotherapy after early versus late diverting stoma closure in low anterior resection for rectal cancer. BMC Cancer.2015;15:923.

79. Alves $A$, Panis $Y$, Lelong $B$ et al. Randomized clinical trial of early versus delayed temporary stoma closure after proctectomy. Br J Surg. 2008;95:693698.

80. Robertson JP, Puckett J, Vather R et al. Early closure of temporary loop ileostomy: a systematic review. Ostomy Wound Manag. 2015;61:50-57.

81. Lasithiotakis K, Aghahoseini A, Alexander D. Is early reversal of defunctioning ileostomy a Shorter, easier and less expensive operation? World J Surg. 2016;40(7):1737-40. 


\section{Archivos de Coloproctología}

Archivos de Coloproctología, 2019; 3(3):05-47 DOI: https://doi.org/10.26754/ojs_arcol/arch_colo.201934001

82. Farag S, Rehman S, Sains P. Early vs delayed closure of loop defunctioning ileostomy in patients undergoing distal colorectal resections: an integrated systematic review and meta- analysis of published randomized controlled trials. Colorectal Dis. 2017;19(12):1050-1057.

83. Klek S, Pisarka M, Milian-Ciesielska K, et al. Early closure of the protective ileostomy after rectal resection should become part of the Enhanced Recovery After Surgery (ERAS) protocol: a randomized, prospective, two-center clinical trial. Wideochir Inne Tech Maloinwzyjne. 2018;13(4):435-441.

84. Fitzgerald PG, Lau GY, Cameron GS. Use of the umbilical site por temporary ostomy: review of 47 cases. J. Pediatr Surg. 1989;24(10):973.

85. Eto K, Omura N, Haruki K. Transumbilical defunctioning ileostomy. A new approach for patients at risks of anastomotic leakage after laparoscopic low anterior resection. Anticancer Res. 2013;33(11):5011-5.

86. Mushava CD, Chandra R, Sansom W, et al. Temporary diverting ileostomy via the umbilicus: a small case series. Int Surg. 2015; 100(3):436-43.

87. Miyo M, Takemasa I, Hata T. Safety and feasibility of umbilical diverting loop ileostomy for patients with rectal tumor. World J Surg. 2017;41(12):32053211.

88. D'Alessandro A, Kari N, Alameh A, et al. Preliminary experience with umbilical stoma in transumbilical single-port colorectal surgery. Tech Coloproctol. 2018;22(4):301-304.

89. Monzón-Abad A, García-Roche C, Martínez.Germán A, et al. A preliminary study of transcaecal ileostomy as an alternative to defunctioning ostomies. Colorectal Dis. 2014;16(2):130-3.

90. Wang J, Ke B, Lin J, et al. Application of a spontaneously closed protective stoma in an ileal pouch-anal anastomosis: a preliminary study. Int J Clin Exp Med. 2015;8(1):1281-1285. 


\section{Archivos de Coloproctología}

Archivos de Coloproctología, 2019; 3(3):05-47

DOI: https://doi.org/10.26754/ojs_arcol/arch_colo.201934001

91. Zhou X, Lin C, Chen W, et al. Completely diverted tube ileostomy compared with loop ileostomy for protection of low colorectal anastomotis: a pilot study. Colorectal Dis. 2014;16(9):O327-31.

92. Hua $\mathrm{H}, \mathrm{Xu} J$, Chen $\mathrm{W}$, et al. Defunctioning cannula ileostomy after lower anterior resection of rectal cancer. Dis Colon Rectum. 2014;57(11):1267-74.

93. Chen $\mathrm{D}$, Zhao $\mathrm{H}$, Huan $\mathrm{Q}$, et al. Application of spontaneously closing cannula ileostomy in laparoscopic anterior resection of rectal cancer. Oncology Letters 2017;14:5299-5306.

94. Zong Z, Zhou T, Jiang Z, et al. Temporary tube stoma versus conventional loop stoma for the protection of a low anastomosis in colorectal surgery: $A$ systematic review and meta-analysis. Am Surg. 2016;82(3):251-8.

95. Sacchi M, Legge PD, Picozzi P. Virtual ileostomy following TME and primary sphincter-saving reconstruction for rectal cancer. Hepatogastroenterology. 2007;54(78):1676-8.

96. Mori L, Vita M, Razzetta F. Ghost ileostomy in anterior resection for rectal carcinoma: is it worthwhile?. Dis Colon Rectum. 2013 Jan;56(1):29-34.

97. Marrosu A, Serventi F, Pulighe F, et al. The "virtual ileostomy" in elective colorectal surgery: Is it useful?. Tech Coloproctol (2014) 18:319-320.

98. Mari FS, Di Cesare T, Novi L. Does ghost ileostomy have a role in the laparoscopic rectal surgery era? A randomized controlled trial. Surg Endoscop 2015;29:2590-7.

99. Ambe PC, Zirngibl H, Möslein G. Routine virtual ileostomy following restorative proctocolectomy for familial adenomatous polyposis. World J Surg. 2018;42(6):1867-71.

100. Kim JH, Kim S, Jung SH. Fecal diverting device for the substitution of defunctioning stoma: preliminary clinical study. Surg Endosc. 2018 Aug 14. 